\title{
The influence of Imre Simon's work in the theory of automata, languages and semigroups
}

\section{Jean-Éric Pin ${ }^{1}$}

Received: 1 January 2019 / Accepted: 12 January 2019 / Published online: 31 January 2019

(c) Springer Science+Business Media, LLC, part of Springer Nature 2019

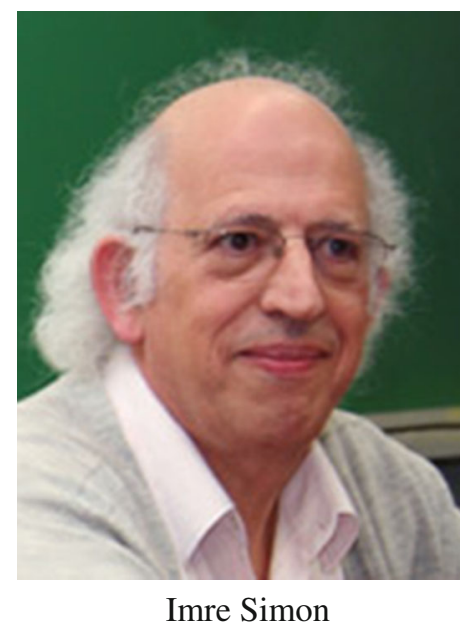

\section{Introduction}

Imre Simon, a Brazilian mathematician and computer scientist, was born in Budapest, Hungary on August 14, 1943. He died in São Paulo, Brazil on August 13, 2009, just a day short of his 66th birthday. More details on his life can be found in the preface to the volume written on the occasion of his 60th birthday (Choffrut and Wakabayashi in Theor Inform Appl 39(1): i-vii, 2005). Among the contributions of this volume, the article Imre Simon: an exceptional graduate student by Thérien (Theor Inform

Communicated by László Márki.

Funded by the DeLTA project (ANR-16-CE40-0007).

$\triangle$ Jean-Éric Pin

Jean-Eric.Pin@irif.fr

1 IRIF, CNRS and Université Paris-Diderot, Case 7014, 75205 Paris Cedex 13, France 
Appl 39(1):297-304, 2005) deserves special mention. The purpose of this tribute is to review three major results due to Simon that had a considerable influence on the development of automata and semigroup theory.

\section{$1 \mathcal{J}$-trivial monoids and their languages}

Let $A$ be a finite alphabet. A language of $A^{*}$ is piecewise testable if it is a Boolean combination of languages of the form $A^{*} a_{1} A^{*} a_{2} \cdots A^{*} a_{k} A^{*}$, where $a_{1}, \ldots, a_{k}$ are letters. In 1972, Simon obtained an elegant characterization of these languages. Recall that a monoid is $\mathcal{J}$-trivial if each of its $\mathcal{J}$-classes is a singleton.

Theorem 1.1 (Simon $[47,48]$ ). A language is piecewise testable if and only if its syntactic monoid is finite and $\mathcal{J}$-trivial.

This result inspired a lot of subsequent research and a number of alternative proofs have been proposed [1,2,23,25,28,29,60,63], not to mention unpublished ones. It was proved seven years after Schützenberger's characterization of star-free languages in terms of aperiodic monoids [45] and these two results, together with Theorem 2.1 below, inspired Eilenberg's varieties theorem [16].

Theorem 1.1 is a key step in the study of concatenation hierarchies initiated by Cohen and Brzozowski [11]. A detailed account of the numerous developments on this topic, including connections with automata, languages and finite model theory can be found in the survey [39]. For this reason, I will omit this important topic and rather focus on some consequences in semigroup theory. For each integer $n>0$, three monoids, $\mathcal{C}_{n}, \mathcal{R}_{n}$ and $\mathcal{U}_{n}$ will serve us as examples of $\mathcal{J}$-trivial monoids.

The monoid $\mathcal{C}_{n}$ consists of all order preserving and extensive functions from $\{1, \ldots, n\}$ to itself. Recall that a transformation $a$ on $\{1, \ldots, n\}$ is order preserving if $p \leqslant q$ implies $p \cdot a \leqslant q \cdot a$ and extensive if for all $p, p \leqslant p \cdot a$.

Let $\mathcal{R}_{n}$ denote the monoid of all reflexive relations on $\{1, \ldots, n\}$. It is convenient to consider $\mathcal{R}_{n}$ as the monoid of Boolean matrices of size $n \times n$ having only entries 1 on the diagonal. For example

$$
\mathcal{R}_{2}=\left\{\left(\begin{array}{ll}
1 & 0 \\
0 & 1
\end{array}\right),\left(\begin{array}{ll}
1 & 1 \\
0 & 1
\end{array}\right),\left(\begin{array}{ll}
1 & 0 \\
1 & 1
\end{array}\right),\left(\begin{array}{ll}
1 & 1 \\
1 & 1
\end{array}\right)\right\}
$$

Finally, $\mathcal{U}_{n}$ is the submonoid of $\mathcal{R}_{n}$ consisting of the upper triangular matrices of $\mathcal{C}_{n}$. The matrices of $\mathcal{U}_{n}$ are called unitriangular. For example,

$$
\mathcal{U}_{3}=\left\{\left(\begin{array}{ccc}
1 & \varepsilon_{1} & \varepsilon_{2} \\
0 & 1 & \varepsilon_{3} \\
0 & 0 & 1
\end{array}\right) \mid \varepsilon_{1}, \varepsilon_{2}, \varepsilon_{3} \in\{0,1\}\right\}
$$

Recall that a monoid $M$ divides a monoid $N$ if $M$ is a quotient of a submonoid of $N$. It is relatively easy to prove the equivalence of Theorems 1.1 and the following result of Straubing [61], but proving one of them is much more difficult.

Theorem 1.2 For each $n>0$, the monoids $\mathcal{C}_{n}, \mathcal{R}_{n}$ and $\mathcal{U}_{n}$ are $\mathcal{J}$-trivial. Moreover, for a finite monoid $M$, the following conditions are equivalent: 
(1) $M$ is $\mathcal{J}$-trivial,

(2) there exists an integer $n>0$ such that $M$ divides $\mathcal{C}_{n}$,

(3) there exists an integer $n>0$ such that $M$ divides $\mathcal{R}_{n}$,

(4) there exists an integer $n>0$ such that $M$ divides $\mathcal{U}_{n}$.

Almeida obtained another proof of Theorem 1.1 as a byproduct of his complete description of the free profinite $\mathcal{J}$-trivial monoid [1]. He proved in particular that the free profinite $\mathcal{J}$-trivial monoid on an $n$-letter alphabet is countable and contains $2^{n}$ idempotents.

Another important consequence of Theorem 1.1 relates $\mathcal{J}$-trivial monoids to ordered monoids. It was first stated and proved by Straubing and Thérien [63]. An ordered monoid is a monoid equipped with a partial order $\leqslant$ compatible with the product: $x \leqslant y$ implies $x z \leqslant y z$ and $z x \leqslant z y$.

Theorem 1.3 A finite monoid is $\mathcal{J}$-trivial if and only if it is a quotient of a finite ordered monoid satisfying the identity $1 \leqslant x$.

Again, it is not very difficult to establish the equivalence of Theorems 1.1 and 1.3. But Straubing and Thérien gave a direct proof of Theorem 1.3 based on the ideal structure of the monoid and a suitable semigroup expansion. Another proof of Theorem 1.3 proposed in [23] makes use of factorisation trees, a notion inspired by Imre Simon's factorisation forests described in Sect. 3.

Theorem 1.3, far from being an isolated result, is actually the prototype of similar covering theorems involving ordered monoids. Recall that a block group is a monoid in which every regular $\mathcal{R}$-class and $\mathcal{L}$-class contains a unique idempotent. Many equivalent definitions can be found for instance in [38]. The following result was first stated in [42, Corollary 9.7], but is a consequence of a series of some deep results obtained in the eighties $[4,5,21,22,24,30]$. See [38] for a survey and related results.

Theorem 1.4 Every finite block group monoid is the quotient of a finite ordered monoid satisfying the identity $1 \leqslant x^{\omega}$.

This result can be relativised to monoids with commuting idempotents but, even in this case, it is a challenging open problem to find a direct proof of this result using the techniques proposed in [63] or in [23].

Simon's original proof [48] relied on the combinatorial study of subwords, one of his favourite topics [58]. He also used the related shuffle operation to solve with Ésik an open problem in language theory [17]. Subwords were later used to describe languages whose syntactic monoids are $p$-groups [16, p. 238]. Another elegant proof of Theorem 1.1 via combinatorics on words was proposed by Klíma [28] and is probably the shortest proof known to date.

The combinatorial study of subwords was initiated by Chen, Fox and Lyndon [9] in a different context. See the survey chapter [33, Chapter 6] written by Sakarovitch and Simon, and [43] for applications to Lie algebras. 


\section{Locally trivial semigroups and graph congruences}

A semigroup $S$ is said to be locally trivial if, for every idempotent $e$ of $S, e S e=e$. Similarly, a semigroup $S$ is said to be locally idempotent and commutative if, for every idempotent $e$ of $S$, the monoid $e S e$ is idempotent and commutative, i.e. a semilattice.

Locally trivial semigroups form a variety of finite semigroups, denoted $\mathbf{L I}$. If $\mathbf{V}$ is a variety of finite monoids, let $\mathbf{V} * \mathbf{L I}$ be the variety generated by all semidirect products of monoids in $\mathbf{V}$ with semigroups in $\mathbf{L I}$.

A language of $A^{+}$is locally testable if it is a Boolean combination of sets of the form $u A^{*}, A^{*} v$ or $A^{*} w A^{*}$ where $u, v, w \in A^{+}$. It it relatively easy to prove that a language is locally testable if and only if its syntactic semigroup belongs to the variety $\mathbf{S L} * \mathbf{L I}$ where SL is the variety of finite semilattices. Quoting Eilenberg [16, p. 222], this is not regarded as a satisfactory solution as it is not clear how to decide membership in this variety. A much better answer was given independently by Brzozowski and Simon [8] and by McNaughton [36], who proved the following result:

Theorem 2.1 A language is locally testable if and only if its syntactic semigroup is locally idempotent and commutative.

In terms of semigroups, a finite semigroup belongs to $\mathbf{S L} * \mathbf{L I}$ if and only if it is locally idempotent and commutative. The proof relies on a result on graph congruences first detailed in Simon's Ph.D. thesis [47]. Given a finite graph $G$, a path congruence is an equivalence relation on the set of paths of $G$ such that:

(1) any two equivalent paths are coterminal (i.e. have the same origin and the same end),

(2) if $p$ and $q$ are equivalent paths, and if $r, p$ and $s$ are consecutive paths, then $r p s$ is equivalent to rqs.

Theorem 2.2 (Simon [47]) Let $\sim$ be a path congruence such that, for every pair of loops $p, q$ around the same state, $p^{2} \sim p$ and $p q \sim q p$. Then two coterminal paths using the same sets of edges are equivalent.

Theorem 2.2 initiated the graph congruence techniques later developed in a series of subsequent papers by Knast [30], Thérien [64], Thérien-Weiss [66], Straubing [62], Jones and Szendrei [26], Jones and Trotter [27], Almeida [3], Pin, Pinguet and Weil [41], Steinberg [59], etc.

Straubing [62] generalized Theorems 2.1 and 2.2 to describe membership in $\mathbf{V} * \mathbf{L I}$ in terms of graph congruences. The modern formulation in terms of categories is due to Tilson [67] (see also [44, p. 279, Theorem 4.8.9]).

Theorem 2.2 is also one of the key ingredients for the algebraic proof of McNaughton's theorem on the equivalence between non deterministic Büchi automata and deterministic Muller automata over infinite words [32].

\section{Factorization forests}

Ramsey's Theorem is frequently used in combinatorics on words to establish the existence of unavoidable regularities in very long words [33, Chapter 4]. Simon's 
factorisation forest theorem is a nested variant of this result. It has become over the years an essential tool in the theory of finite semigroups.

A factorisation forest is a function $d$ that associates to every word $x$ of length $\geqslant 2$ a factorisation $d(x)=\left(x_{1}, \ldots, x_{n}\right)$ of $x$ with $n \geqslant 2$ such that $x_{1}, \ldots, x_{n}$ are nonempty words. The integer $n$ is the degree of the factorisation.

Let $S$ be a finite semigroup and let $\varphi: A^{+} \rightarrow S$ be a morphism. A factorisation forest $d$ is $\varphi$-Ramseyan if, for every word $x$ of length $\geqslant 2, d(x)$ is either of degree 2 or there exists an idempotent $e$ of $S$ such that $d(x)=\left(x_{1}, \ldots, x_{n}\right)$ and $\varphi\left(x_{1}\right)=\varphi\left(x_{2}\right)=$ $\ldots=\varphi\left(x_{n}\right)=e$ for $1 \leqslant i \leqslant n$.

Simon first stated his factorisation forest theorem in the technical report [50], which was subsequently published in [52,53]. He later gave a different proof in [55].

Theorem 3.1 (Simon [50]) Let $\varphi: A^{+} \rightarrow S$ be a morphism onto a finite semigroup. Then there exists a factorisation forest of height $\leqslant 9|S|$ which is $\varphi$-Ramseyan.

The bound $9|S|$ was subsequently improved to $3|S|$ in [13] and to the optimal bound $3|S|-1$ in [31]. Simon was led to his factorisation forest theorem in connection with his research on three topics: Brown's theorem on locally finite semigroups, the limitedness problem for the tropical semiring $[34,49,51,56]$ and the star-height problem. Despite their importance, I will not develop further these topics, which are already covered in the historical survey [40].

The term tropical was given in honour of Imre Simon, who gave credit to Christian Choffrut in [51], but further investigation among the members of the French school suggests that the term was originally coined by Schützenberger. The term has become standard since the success of tropical geometry.

Simon's factorisation forest theorem has been extended by Colcombet to infinite words [13] and to trees [12]. It is also crucial in the characterization of polynomial languages [42]. Bojanczyck [6] wrote a survey of applications of factorisation forests to fast string algorithms and transducers. There are aspects of Simon's work that remain to be explored. For instance, very recently an unambiguous version of the Factorization Theorem has been announced in [15,18].

The reader is referred to the recent survey by Colcombet [14] for more information on Simon's forest factorisation theorem.

\section{Conclusion}

Due to space constraints, several areas studied by Imre Simon had to be omitted, notably nondeterministic complexity of finite automata [54], compression and entropy [20], string matching algorithms [57]. However, I would like to briefly mention Simon's research on the Burnside problem for semigroups. Simon's contribution to this question is reported in the survey [37] coauthored with do Lago.

The general problem concerns the free Burnside monoid satisfying $x^{n}=x^{n+m}$, but the results of Green and Rees [19] and McLean [35] essentially reduce the problem for $n=1$ to the case $n=0$, that is, the Burnside problem for groups. Simon addressed in 1970 what he thought to be the easiest remaining case: the free Burnside monoid satisfying $x^{2}=x^{3}$. Unfortunately for him, it turns out to be the hardest case, which 
is not yet completely understood. Simon rediscovered the fact that this monoid was infinite, a result previously obtained by Brzozowski et al. [7], but also extended some properties of the free bands to the free Burnside monoids. He never published his results [46], but later passed the problem on to his best student, Alair Pereira do Lago, who obtained the beautiful results reported in the surveys [37] and [40, Section 6].

Imre Simon's work had a tremendous impact on subsequent research and considerable influence in semigroup theory, automata, formal languages, combinatorics on words and finite model theory. Ten years after his death, his work is still at the heart of current research.

Acknowledgements I would like to thank László Márki and Norman Reilly for their help during the preparation of this article.

\section{References}

1. Almeida, J.: Implicit operations on finite $\mathcal{J}$-trivial semigroups and a conjecture of I. Simon. J. Pure Appl. Algebra 69(3), 205-218 (1991)

2. Almeida, J.: Finite Semigroups and Universal Algebra. Series in Algebra, vol. 3. World Scientific, Singapore (1994)

3. Almeida, J.: A syntactical proof of locality of DA. Int. J. Algebra Comput. 6(2), 165-177 (1996)

4. Ash, C.J.: Finite semigroups with commuting idempotents. J. Aust. Math. Soc. Ser. A 43(1), 81-90 (1987)

5. Ash, C.J.: Inevitable graphs: a proof of the type II conjecture and some related decision procedures. Int. J. Algebra Comput. 1(1), 127-146 (1991)

6. Bojańczyk, M.A: Factorization forests, in developments in language theory. In: Diekert, V., Nowotka, D. (eds.) Proceedings of 13th International Conference, DLT 2009, Lecture Notes in Computer Science, vol. 5583 , pp. 1-17. Springer, Berlin (2009)

7. Brzozowski, J.A., Čulík II, K., Gabrielian, A.: Classification of noncounting events. J. Comput. Syst. Sci. 5, 41-53 (1971)

8. Brzozowski, J.A., Simon, I.: Characterizations of locally testable events. Discrete Math. 4, 243-271 (1973)

9. Chen, K.-T., Fox, R .H., Lyndon, R .C.: Free differential calculus. IV. The quotient groups of the lower central series. Ann. Math. 68, 81-95 (1958)

10. Choffrut, C., Wakabayashi, Y.: Preface [Imre Simon, the tropical computer scientist]. Theor. Inform. Appl. 39(1), i-vii (2005)

11. Cohen, R.S., Brzozowski, J.A.: Dot-depth of star-free events. J. Comput. Syst. Sci. 5(1), 1-16 (1971)

12. Colcombet, T.: A combinatorial theorem for trees. In: Arge, L., Cachin, C., Jurdzinski, T., Tarlecki, A. (eds.) Proceedings of 34th International Conference on Automata, Languages, and Programming (ICALP). Lecture Notes in Computer Science, vol. 4596, pp. 901-912. Springer, New York (2007)

13. Colcombet, T.: Factorisation forests for infinite words. In: Csuhaj-Varjú, E., Ésik, Z. (eds.) Fundamentals of Computation Theory, 16th International Symposium: FCT '07. Lecture Notes in Comput. Sci. vol. 4639, pp. 226-237. Springer, New York (2007)

14. Colcombet, T.: The factorisation forest theorem. In: Pin, J.-É. (ed.) Handbook of Automata Theory, vol. I: Theoretical Foundations, European Mathematical Society, Zürich (2019)

15. Dave, V., Gastin, P., Krishna, S.N.: Regular transducer expressions for regular transformations. In: Dawar, A., Grädel, E. (eds.) Proceedings of 33rd IEEE Symposium on Logic in Computer Science, pp. 315-324 (2018)

16. Eilenberg, S.: Automata, languages, and machines. Vol. B, Academic Press [Harcourt Brace Jovanovich Publishers], New York, 1976. With two chapters ("Depth decomposition theorem" and "Complexity of semigroups and morphisms") by Bret Tilson, Pure and Applied Mathematics, Vol. 59, (1976)

17. Ésik, Z., Simon, I.: Modeling literal morphisms by shuffle. Semigroup Forum 56(2), 225-227 (1998)

18. Gastin, P., Krishna, S.N.: Unambiguous Forest Factorization, CoRR abs/1810.07285 (2018)

19. Green, J.A., Rees, D.: On semi-groups in which $x^{r}=x$. Proc. Camb. Philos. Soc. 48, 35-40 (1952) 
20. Hansel, G., Perrin, D., Simon, I.: Compression and entropy. In: Finkel, A., Jantzen, M. (eds.) STACS 92, Proceedings of 9th Symposium Theoretical Aspects of Computer Science. Lecture Notes in Computer Science, vol. 577, pp. 515-528. Springer, Berlin (1992)

21. Henckell, K.: Blockgroups = powergroups: a consequence of Ash's proof of the Rhodes type II conjecture. In: Monash Conference on Semigroup Theory (Melbourne, 1990), pp. 117-134, World Sci. Publ., River Edge (1991)

22. Henckell, K., Margolis, S .W., Pin, J.-É., Rhodes, J.: Ash's type II theorem, profinite topology and Mal'cev products. I. Int. J. Algebra Comput. 1(4), 411-436 (1991)

23. Henckell, K., Pin, J.-É.: Ordered monoids and $\mathcal{J}$-trivial monoids. In: Algorithmic Problems in Groups and Semigroups (Lincoln. NE, 1998), Trends Mathematicla, pp. 121-137. Birkhäuser Boston, Boston (2000)

24. Henckell, K., Rhodes, J.: The theorem of Knast, the $P G=B G$ and type-II conjectures. In: Monoids and Semigroups with Applications (Berkeley, CA, 1989), pp. 453-463. World Scientific Publication, River Edge (1991)

25. Higgins, P.M.: A proof of Simon's theorem on piecewise testable languages. Theoret. Comput. Sci. 178(1-2), 257-264 (1997)

26. Jones, P.R., Szendrei, M.B.: Local varieties of completely regular monoids. J. Algebra 150(1), 1-27 (1992)

27. Jones, P.R., Trotter, P.G.: Locality of DS and associated varieties. J. Pure Appl. Algebra 104(3), 275301 (1995)

28. Klíma, O.: Piecewise testable languages via combinatorics on words. Discrete Math. 311(20), 21242127 (2011)

29. Klíma, O., Polák, L.: Alternative automata characterization of piecewise testable languages. In: Developments in Language Theory. Lecture Notes in Computer Sciecne, vol. 7907,, pp. 289-300. Springer, Heidelberg (2013)

30. Knast, R.: Some theorems on graph congruences. RAIRO Inform. Théor. 17(4), 331-342 (1983)

31. Kufleitner, M.: The height of factorization forests. In: Ochmanski, E., Tyszkiewicz, J. (eds.) Proceedings of 33rd Symposium, Mathematical Foundations of Computer Science 2008. Lecture Notes in Computer Science, vol. 5162, pp. 443-454. Springer, Berlin (2008)

32. Le Saec, B., Pin, J.-É., Weil, P.: Semigroups with idempotent stabilizers and applications to automata theory. Int. J. Algebra Comput. 1, 291-314 (1991)

33. Lothaire, M.: Combinatorics on Words, Encyclopedia of Mathematics and its Applications, vol. 17. Cambridge University Press, Cambridge (1983)

34. Mandel, A., Simon, I.: On finite semigroups of matrices. Theoret. Comput. Sci. 5(2), 101-111, (1977/78)

35. McLean, D.: Idempotent semigroups. Am. Math. Mon. 61, 110-113 (1954)

36. McNaughton, R.: Algebraic decision procedures for local testability. Math. Syst. Theory 8(1), 60-76 (1974)

37. Pereira Do Lago, A., Simon, I.: Free Burnside semigroups. Theor. Inform. Appl. 35(6), (2001) 579-595 (2002). A tribute to Aldo de Luca

38. Pin, J.-É.: $P G=B G$, a success story. In: Fountain, J. (ed.) Semigroups, Formal Languages and Groups (York, 1993). NATO Advanced Science Institutes Series C: Mathematical and Physical Science, vol. 466, pp. 33-47. Kluwer, Dordrecht (1995)

39. Pin, J.-É.: The dot-depth hierarchy, 45 years later. In: Konstantinidis, S., Moreira, N., Reis, R., Jeffrey, S. (eds.) The Role of Theory in Computer Science-Essays Dedicated to Janusz Brzozowski, pp. 177-202. Word Scientific (2017)

40. Pin, J.-É.: Open Problems about Regular Languages, 35 Years Later. In: Konstantinidis, S., Moreira, N., Reis, R., Jeffrey, S. (eds.) The Role of Theory in Computer Science, Essays dedicated to Janusz Brzozowski, pp. 153-176. Word Scientific (2017)

41. Pin, J.-É., Pinguet, A., Weil, P.: Ordered categories and ordered semigroups. Commun. Algebra 30, 5651-5675 (2002)

42. Pin, J.-É., Weil, P.: Polynomial closure and unambiguous product. Theory Comput. Syst. 30(4), 383422 (1997)

43. Reutenauer, C.: Free Lie algebras, London Mathematical Society Monographs. New Series, vol. 7. The Clarendon Press, Oxford University Press, New York (1993). Oxford Science Publications

44. Rhodes, J., Steinberg, B.: The $q$-theory of Finite Semigroups, Springer Monographs in Mathematics. Springer, New York (2009) 
45. Schützenberger, M.-P.: On finite monoids having only trivial subgroups. Inf. Control 8, 190-194 (1965)

46. Simon, I.: Notes on non-counting languages of order 2. Manuscript

47. Simon, I.: Hierarchies of Events with Dot-Depth One, PhD thesis, University of Waterloo, Waterloo, Ontario, Canada, (1972)

48. Simon, I.: Piecewise testable events. In: Brackage, H. (ed.) Proceedings of 2 nd GI Conference. Lecture Notes in Computer Science vol. 33, pp. 214-222. Springer, Berlin (1975)

49. Simon, I.: Limited subsets of a free Monoid. In: Proceedings of 19 th Annual Symposium on Foundations of Computer Science, Piscataway, NJ, pp. 143-150. IEEE (1978)

50. Simon, I.: Factorization Forests of Finite Height, Rap. Tech., Report 87-73, Laboratoire d'Informatique Théorique et Programmation, Paris, (1987)

51. Simon, I.: Recognizable sets with multiplicities in the tropical semiring. In: Chytil, M., Janiga, L., Koubek, V. (eds.) Proceedings of 13th Symposium, Mathematical Foundations of Computer Science. Lecture Notes in Computer Science, vol. 324, pp. 107-120. Springer (1988)

52. Simon, I.: Properties of factorization forests. In: Proceedings of Formal Properties of Finite Automata and Applications, Ramatuelle, France, Berlin. Lecture Notes in Computer Science, vol. 386, pp. 65-72. Springer (1989)

53. Simon, I.: Factorization forests of finite height. Theoret. Comput. Sci. 72(1), 65-94 (1990)

54. Simon, I.: The nondeterministic complexity of a finite automaton. In: Mots, mélanges offerts à, Schützenberger, M.P., Lothaire, M. (eds.) pp. 384-400. Hermès, Paris, Lang. Raison. Calc. (1990)

55. Simon, I.: A short proof of the factorization forest theorem. In: Nivat, M., Podelski, A. (eds.), Tree Automata and Languages (Le Touquet, 1990), Amsterdam. Study Computer Science Artificial Intelligence vol. 10, pp. 433-438. North-Holland (1992)

56. Simon, I.: On semigroups of matrices over the tropical semiring. RAIRO Inform. Théor. Appl. 28(3-4), 277-294 (1994)

57. Simon, I.: String matching algorithms and automata. In: Results and Trends in Theoretical Computer Science (Graz, 1994). Lecture Notes in Computer Science, vol. 812, pp. 386-395. Springer, Berlin (1994)

58. Simon, I.: Words distinguished by their subwords. In: Proceedings of WORDS'03. TUCS General Publication vol. 27, pp. 6-13. Turku Center Computer Science, Turku (2003)

59. Steinberg, B.: A structural approach to the locality of pseudovarieties of the form $\mathbf{L H}(\mathrm{M}) \mathbf{V}$. Internat. J. Algebra Comput. 20(2), 307-318 (2010)

60. Stern, J.: Characterizations of some classes of regular events. Theoret. Comput. Sci. 35(1), 17-42 (1985)

61. Straubing, H.: On finite $\mathcal{J}$-trivial monoids. Semigroup Forum 19(2), 107-110 (1980)

62. Straubing, H.: Finite semigroup varieties of the form $V * D$. J. Pure Appl. Algebra 36(1), 53-94 (1985)

63. Straubing, H., Thérien, D.: Partially ordered finite monoids and a theorem of I. Simon. J. Algebra 119(2), 393-399 (1988)

64. Thérien, D.: Two-sided wreath product of categories. J. Pure Appl. Algebra 74(3), 307-315 (1991)

65. Thérien, D.: Imre Simon: an exceptional graduate student. Theor. Inform. Appl. 39(1), 297-304 (2005)

66. Thérien, D., Weiss, A.: Graph congruences and wreath products. J. Pure Appl. Algebra 36(2), 205-215 (1985)

67. Tilson, B.: Categories as algebra: an essential ingredient in the theory of monoids. J. Pure Appl. Algebra 48(1-2), 83-198 (1987)

Publisher's Note Springer Nature remains neutral with regard to jurisdictional claims in published maps and institutional affiliations. 